\title{
Simple Specimen Preparation Method for In Situ Heating Experiments
}

\author{
Qingxiao Wang ${ }^{1}$, Jinguo Wang ${ }^{1}$ and Moon J. Kim ${ }^{1}$ \\ ${ }^{1}$ Dept. of Materials Science and Engineering, The University of Texas at Dallas, Richardson, TX
}

With the development of spherical aberration-corrected transmission electron microscope/scanning transmission electron microscope (Cs-TEM/STEM), atomic resolution can be achieved from routine TEM/STEM experiments. The in-situ techniques of the chemical reaction, phase transition, electrical and mechanical properties at atomic level have received great interests. Recently several in-situ TEM holders and MEMS based chips are commercialized which greatly improve the capability of in-situ study down to the atomic scale. However, specimen preparation technique is a critical limiting factor for the in-situ study.

Most of publications on in-situ heating experiments are focused on nanomaterials, such as nanoparticles and nanowires, since the specimen can be prepared by a solution suspension method. For the characterization of bulk materials and thin film samples, focused ion beam (FIB) method has been widely used and some researchers have developed techniques to transfer the TEM lamella onto the in-situ heating chip using in-situ lift out (ISLO) [1] or ex-situ lift out (ESLO) methods [2]. Recently two-dimensional (2D) nanomaterials including graphene, hexagonal boron nitride (h-BN), transition-metal dichalcogenides (TMDs) have received great attentions because of their unique electronic, optical, and chemical properties. To date, mechanical exfoliation is still the most efficient way to create the clean atomically thin flakes for TEM analysis. Both wet and dry transfer methods have been developed to transfer the 2D materials on the in-situ heating chip for phase transition and thermal stability study [3,4]. For the wet transfer method, PMMA is generally used as a sacrificial film during the transfer process, followed by acetone washing to dissolve it. But PMMA residue is a big issue for making clean TEM specimen. Although baking the sample at elevated temperature under vacuum or ambient $\left(\mathrm{Ar} / \mathrm{H}_{2}\right)$ condition can remove PMMA residue, it is not applicable for those materials with phase transition at elevated temperature. For the all-dry transfer method, clean sample surface can be achieved. But the PDMS peeling procedure may cause the thin and fragile heating membrane broken. To overcome the drawbacks of these methods, we demonstrate a simple way to transfer the TMDs flakes on the heating chip by combining the mechanical exfoliation and FIB ISLO methods.

Silicon substrate with a $90 \mathrm{~nm}$ silicon oxide coating layer is cleaned by acetone followed by an $\mathrm{O}_{2}$ plasma treatment for $10 \mathrm{~min}$ to create a hydrophilic surface on $\mathrm{SiO}_{2}$. Atomically thin $\mathrm{MoTe}_{2}$ flakes are peeled off from natural bulk crystal using scotch tape. The tape with $\mathrm{MoTe}_{2}$ flakes is brought into contact with $\mathrm{Si}$ substrate and some flakes are transferred onto the $\mathrm{SiO}_{2}$ surface using the capillary force between $\mathrm{MoTe}_{2}$ flakes and $\mathrm{SiO}_{2}$ surface. In order to create freestanding $\mathrm{MoTe}_{2}$ flakes which will be used for the later ISLO, a Quantifoil TEM grid is placed on top of Si substrate with its carbon side facing the substrate. A drop of acetone is placed on the Quantifoil TEM grid and dried quickly in air, thus the gap between the $\mathrm{MoTe}_{2}$ flakes and carbon film on the grid is removed. The $\mathrm{SiO}_{2}$ layer can be etched and removed by placing the $\mathrm{Si}$ substrate into $1 \mathrm{M} \mathrm{KOH}$ solution, so the $\mathrm{MoTe}_{2}$ flakes are transferred on the Quantifoil TEM grid due to strong capillary force between the carbon film and $\mathrm{MoTe}_{2}$ flakes. Optical microscope or SEM can be used to locate the atomically thin $\mathrm{MoTe}_{2}$ flakes on the grid. Figure 1(a) shows the SEM image of a MoTe $\mathrm{Flake}$ transferred with atomically thin edges.

Figure 1(b) shows the SEM image of the mask made from mechanical polishing a TEM sample by FIB. The mask is used to protect the $\mathrm{MoTe}_{2}$ flake from ion beam damage during ISLO. In Figure 1(c), the mask is transferred and overlaid on the $\mathrm{MoTe}_{2}$ flake with the help of Omniprobe needle. The four corners of the mask are fixed to the Quantifoil carbon film by ion beam assisted Pt deposition. Then ion beam milling is performed to isolate the $\mathrm{MoTe}_{2}$ flake and mask from the Quantifoil TEM grid. Now, the $\mathrm{MoTe}_{2}$ flake under 
the mask is attached on the Omniprobe needle and ready for transfer onto the in-situ heating chip. After carefully aligning the mask with the window of the in-situ heating chip, the $\mathrm{MoTe}_{2}$ flake approaches the heating membrane by gently lowering the Omniprobe needle. The same procedure is applied to connect the four corners with $\mathrm{Ga}$ ion beam assisted $\mathrm{Pt}$ deposition and the $\mathrm{MoTe}_{2}$ flake is fixed on the membrane. Ga ion beam is used to cut off the four thin arms of the mask to release it from the heating chip and the Omniprobe needle with the mask is retracted. Only $\mathrm{MoTe}_{2}$ flakes on a supporting carbon film is left on the heating chip. The mask on the Omniprobe needle can be mounted to a safe place for future use. Figure 1(d) shows the SEM image of the $\mathrm{MoTe}_{2}$ flake on the heating chip ready for in-situ experiments. Due to the existence of the mask over the $\mathrm{MoTe}_{2}$ flake, the area of interest is protected during the whole transfer process. There is no Ga ion beam induced damage to the sample, and the contamination has also been minimized during $\mathrm{Pt}$ deposition. A clean sample surface can be achieved for the in-situ heating experiment. Figure 2(a) and (b) shows STEM-HAADF image taken at room temperature. Clearly, we can confirm the cleanness and pristine nature of the transferred specimen. Furthermore, the transferred specimen by this method has been successfully subjected to the in-situ heating experiment as shown in Figure 2(c) and (d) and the result will be published in detail. [5]

References:

[1] Martial Duchamp et al, Microsc. Microanal. 20, (2014) 1638-1645.

[2] Lucille A. Giannuzzi et al, Microsc. Microanal. 21, (2015) 1034-1048.

[3] Hai Li et al, ACS Nano 8 (7), (2014) 6563-6570.

[4] Andres Castellanos-Gomez et al, 2D Materials 1, (2014) 011002.

[5] This work is supported in part by the Center for Low Energy Systems Technology (LEAST), one of six centers supported by the STARnet phase of the Focus Center Research Program (FCRP), a Semiconductor Research Corporation program sponsored by MARCO and DARPA. It is also supported by the SWAN Center, a SRC center sponsored by the Nanoelectronics Research Initiative and NIST.

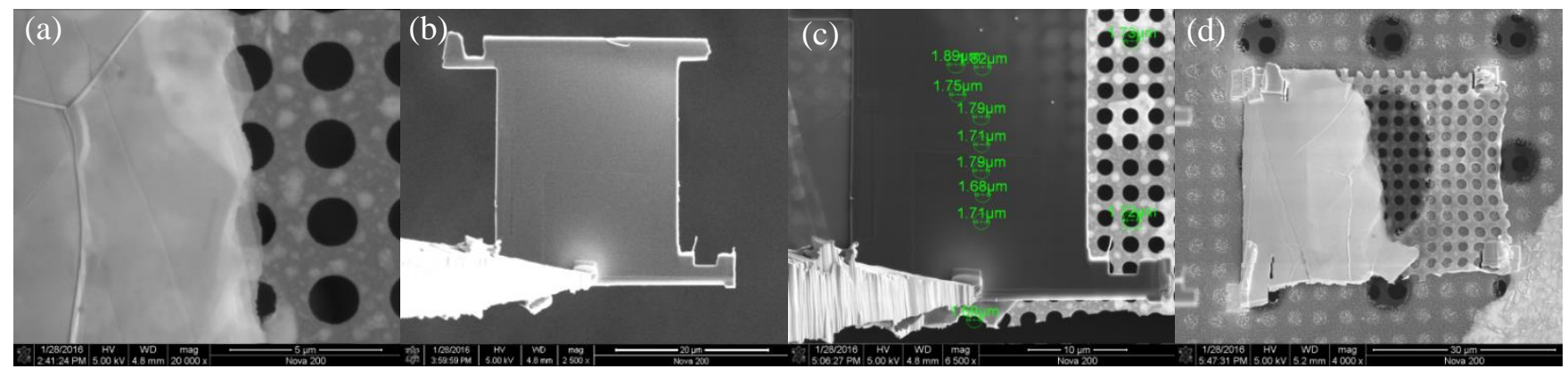

Figure 1. (a) SEM image of $\mathrm{MoTe}_{2}$ flake. (b) SEM image of the mask attached on Omniprobe needle. (c) SEM image of the mask overlay on the $\mathrm{MoTe}_{2}$ flake. The locations of some thin edges have been marked by green circles. (d) SEM image of the $\mathrm{MoTe}_{2}$ flake on the heating chip for in-situ experiment.
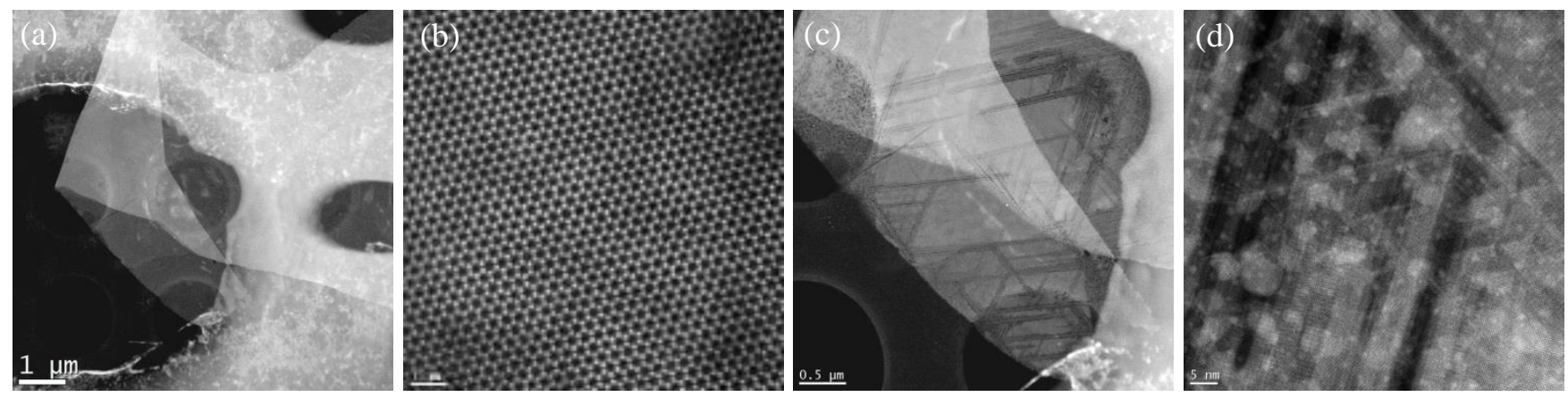

Figure 2. (a) Low magnification and (b) High resolution STEM-HAADF images of a $\mathrm{MoTe}_{2}$ flake taken at room temperature. (c) Low magnification and (d) High resolution HAADF images of the MoTe2 flake taken at $400^{\circ} \mathrm{C}$. 